\title{
Lactancia materna y caries de la infancia temprana
}

\author{
Mario Alberto Maldonado Ramírez ${ }^{1}$ \\ Alejandra González Hernández², \\ Enrique E. Huitzil Muñoz ${ }^{3}$ \\ Hilda Isassi Hernández $z^{4}$ \\ Ricardo Reyes Flores ${ }^{5}$
}

\section{Resumen}

La lactancia materna es una práctica natural destinada a favorecer el crecimiento y desarrollo del lactante, sin embargo, a pesar de que proporciona inmunidad contra ciertos patógenos, se cree que favorece el proceso de la caries dental. Objetivo: El propósito del presente estudio fue comprobar si la lactancia materna contribuye en la aparición de caries dental de la primera infancia. Materiales y Métodos: El presente es un estudio transversal analítico. La muestra incluyó a 164 menores cuyas edades oscilaron entre 6 y 48 meses de edad, divididos en 3 grupos: Lactancia Materna exclu- siva (GRUPO 1), Lactancia combinada (GRUPO 2) y Lactancia exclusiva con biberón (GRUPO 3). Se aplicó una prueba de correlación de Pearson utilizando el programa SPSS 18. Resultados: Se identificó una correlación fuertemente positiva entre el grupo de lactancia materna exclusiva e individuos libres de caries $(\mathrm{p}=.000)$. Conclusión. La lactancia materna durante los primeros meses de vida parece ofrecer una protección contra la caries dental.

Palabras clave: Caries de la infancia temprana, Lactancia materna, Caries infantil, Patrones de alimentación infantil.

\section{Artigos Originais}

\section{Aleitamento materno e cárie na primeira infancia}

\section{Resumo}

A amamentação é uma prática natural destinada a promover o crescimento e desenvolvimento das crianças. Embora ela auxilie na imunidade contra determinados patógenos, se acredita que possa favorecer o processo de cárie dentária. Objetivo: $\mathrm{O}$ objetivo deste estudo foi determinar se o aleitamento materno contribui para o desenvolvimento da cárie dentária na primeira infân- cia. Materiais e métodos: $\mathrm{O}$ presente estudio é um transversal analitico. A amostra consistiu de 164 crianças entre 6 e 48 meses de idade, divididas em 3 grupos: Aleitamento materno exclusiv (GRUPO 1), aleitamento combinad (GRUPO 2) e alimento exclusivo con mamadeira(GRUPO 3). O teste de correlação de Pearson foi aplicado usando SPSS 18. Resultados: Uma forte correlação positiva entre o grupo de aleitamento materno exclusivo e individuos livres de cárie foi 
observada ( $p=0,000)$. Conclusão. O aleitamento materno durante os primeiros meses de vida oferece proteção contra a cárie dentária.
Palavras-chave: Cárie da primeira infância, Aleitamento Materno, Cárie em crianças, padrões de alimentação infantil.

\section{Original Articles}

\section{Breastfeeding and early childhood caries}

\begin{abstract}
Breastfeeding is a natural practice designed to promote the growth and development of infants, however, although it provides immunity against certain pathogens, is believed to favor the process of tooth decay. Objective: The purpose of this study was to determine whether breastfeeding contributes to the development of dental caries in early childhood. Materials and methods: The present is a transversal analytical study. The sample consisted of 164 children between 6 and 48 months old, divided
\end{abstract}

into 3 groups: exclusive breastfeeding (GROUP $1)$, combined breastfeeding (GROUP 2) and exclusive bottle feeding (GROUP 3). a test Pearson correlation was applied using SPSS 18 program. Results: A strong positive correlation between the group of exclusive breastfeeding and caries-free individuals $(p=.000)$ was identified. Conclusion Breastfeeding during the first months of life provides protection against tooth decay.

Key words: Early childhood caries, breastfeeding, Childhood caries, Infant feeding patterns.

\section{Introducción:}

La caries dental es considerada una de las enfermedades más comunes en la infancia, de acuerdo con Setiawati (2006) ${ }^{1}$ y Guerrero (2011), ${ }^{2}$ la caries se puede desarrollar de inmediato tan pronto como el diente entre en erupción en la cavidad oral. En estudios de calidad de vida se ha reportado que la caries dental es una condición que llega a perjudicar el desarrollo integral del niño, pues en estados avanzados ocasiona procesos infecciosos, problemas estéticos, fonéticos y dificulta la masticación. ${ }^{3-10}$ En México, la prevalencia de caries dental en niños supera cualquier otra condición de salud. Según cálculos obtenidos, seis de cada diez niños padecen caries dental a la edad de seis años. De acuerdo con el SIVEPAB (Sistema de Vigilancia Epidemiológica de Patologías Bucales) en los casos registrados por Instituciones del sector salud como: Instituto de Seguro de Salud al Servicio de los Trabajadores del Estado, Desarrollo Integral de la Familia, Secretaria de la Defensa Nacional y Secretaría de Marina, las edades osci- lan entre los 2 y 19 años, el grupo de 2 a 4 años muestra un índice ceo que va de 2.4 a 4.3 y ésta tendencia persiste con la edad y el recambio de dentición primaria a permanente, ya que el grupo de 6 a 19 años de edad muestra un índice CPOD que inicia con 0.1 a los 6 años y aumenta hasta un CPOD de 7 a los 19 años. ${ }^{11,12,13}$ Este comportamiento es similar al de otros países, en donde la prevalencia y gravedad de la caries de la primera infancia es bastante elevada; ${ }^{8-14}$ además, la caries dental es considerada una enfermedad infecciosa inducida por la dieta. ${ }^{15-20} \mathrm{El}$ "Fondo de las Naciones Unidas para la Infancia", recomienda la leche materna como el único alimento que el lactante necesita durante sus primeros 6 meses de vida. ${ }^{21} \mathrm{~A}$ éste respecto, Maldonado y col. (2010), ${ }^{22}$ consideran a la lactancia materna, como un factor protector en el desarrollo de la caries temprana. Esto ya había sido señalado anteriormente por varios investigadores, afirmando que: 1 ) Los niños que son alimentados con leche materna, tienen una mayor probabilidad de encontrarse libre de caries. 2) La ingesta de leche materna por 21 meses no predis- 
pone a la aparición de caries dental. 3) Niños sin historia de amamantamiento materno, tienen $84 \%$ más riesgo de adquirir caries dental. 4) En un estudio in-vitro se confirma que la leche materna es menos cariogénica que la glucosa y la sacarosa, determinando que la lecha materna no causa la descalcificación del esmalte cuando se encuentra expuesto a ella por 12 semanas; sin embargo, si se le adiciona sacarosa, el desarrollo de la caries se presenta después de 3 semanas. ${ }^{23-28}$

Por otra parte, otros estudios consideran que la leche materna administrada de una manera prolongada puede ser perjudicial y desencadenar la aparición de la caries dental, ${ }^{15-18}$ al igual que la combinación del biberón y seno materno nocturno son considerados factores de riesgo para la aparición de caries de la primera infancia. ${ }^{15,22,}$ ${ }^{24,25}$ Sin embargo, Dye y col. $(2004)^{26}$, opinan que las pruebas son insuficientes y atribuyen el desarrollo de la caries temprana a factores como: 1) consumo de jugos y refrescos, 2) aparición temprana de Streptococcus mutans, 3) higiene bucal deficiente; y en forma indirecta: 4) estrato socioeconómico y 5) nivel bajo de educación de los padres. ${ }^{9,10,29-42}$

El objetivo del presente estudio fue comparar los diferentes patrones de alimentación relacionados con la lactancia materna y su asociación con la aparición de caries dental.

\section{Materiales y métodos}

Para llevar a cabo la investigación se diseñó un estudio transversal analítico donde se estudiaron la variable de interés (tipo de alimentación) para confirmar su posible asociación con el efecto (caries de la infancia temprana).

Conformaron la muestra 164 menores de 6 a 48 meses de edad que acudieron a 9 guarderías de las ciudades de Tampico y Madero, Tamaulipas, México. Los niños se agruparon de acuerdo a la alimentación recibida desde su nacimiento: GRUPO 1, los que recibieron alimentación materna exclusivamente; GRUPO 2, los que recibieron alimentación materna combinada con biberón y GRUPO 3, los que recibieron alimentación por biberón exclusivamente. Todos ellos fueron examinados en el consultorio de la guardería por estudiantes de segundo año del postgrado de odontopediatría para diagnosticar caries dental de acuerdo a los criterios de la Organización Mundial de Salud (OMS) y obtener el índice de higiene oral simplificado adaptado a la dentición primaria, en caso de ausencia de uno de los dientes seleccionados para el examen, se consideró el inmediato más cercano del mismo cuadrante. Los examinadores fueron calibrados por un representante de la OMS, obteniendo un coeficiente de concordancia de 0,80 .

Para evaluar si otros factores predisponentes pudieran estar presentes se exploró a los padres o tutores de los menores y se registraron su índice $\mathrm{CPO}$, índice de higiene oral simplificado (Green y Vermillion) y el máximo grado académico obtenido hasta el momento del estudio.

Previo al inicio del estudio, se obtuvo el consentimiento informado para el examen intraoral, tanto del menor como del padre, madre o tutor. Para la recolección de la información, se entregó a la persona responsable de la alimentación del menor un cuestionario con las variables a ser estudiadas: lactancia materna exclusiva, alimentación combinada con biberón, alimentación con biberón exclusivamente así como la edad en meses del infante en que inició la alimentación complementaria. Para el registro de dientes cariados se realizó examen de cavidad oral siguiendo las recomendaciones de la OMS para obtener el índice de caries ceo.

La información se registró en el formato recolector de datos.

Para el presente estudio se obtuvieron medias y correlaciones de las variables de estudio aplicando una prueba de correlación de Pearson con un nivel de significancia de 0.05 , utilizando el programa SPSS 18.

\section{Resultados}

De los 164 encuestados, 81 de ellos fueron niños $(50.6 \%)$ y 83 niñas $(49.4 \%)$ de 6 a 48 meses de edad. 


\begin{tabular}{|ccccc|}
\hline & Total & $\begin{array}{c}\text { Lactancia } \\
\text { Materna }\end{array}$ & Combinada & Biberón \\
\hline N & 164 & 10 & 121 & 33 \\
\hline Porcentaje & $100 \%$ & $6,09 \%$ & $73,70 \%$ & 20,10 \\
\hline
\end{tabular}

Tabla 1. Distribución por estratos.

Table 1. Distribution by stratum.

Se identificaron las medias de las variables del estudio en relación al índice ceo del menor. Encontrando a 10 menores que se ubicaron en el GRUPO 1 con una media de 11 meses de duración, la alimentación complementaria inicio a los 4.7 meses, este grupo obtuvo una media del índice ceo igual a 0,10 lo que permitió ubicarlos en la categoría de prevalencia de caries "muy bajo".

En los 121 menores que formaron el GRUPO 2 se detecto una media de 3 meses de duración de lactancia materna, en estos menores examinados el inicio de la alimentación complementaria fue a los 5 meses, este grupo presento un índice ceo igual a 0,36 aunque el valor es ligeramente mayor que el del GRUPO 1, se encontraron dentro de la categoría de prevalencia de caries "muy bajo" al igual que el GRUPO 1.

El último grupo lo formaron 33 menores que se ubicaron en el GRUPO 3 ellos iniciaron la ingesta de alimentación complementaria a los 5.5 meses de edad y presentaron un índice ceo igual a 0,33 también se ubicaron en la categoría de prevalencia de caries "muy bajo" similar a los grupos anteriores.

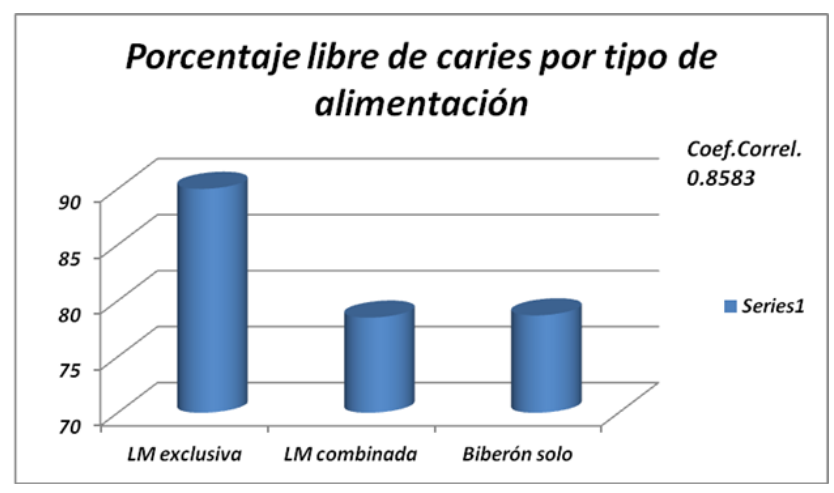

Figura 2. Porcentaje libre de caries por tipo de alimentación. Figure 2. Percentage of caries-free feed type.

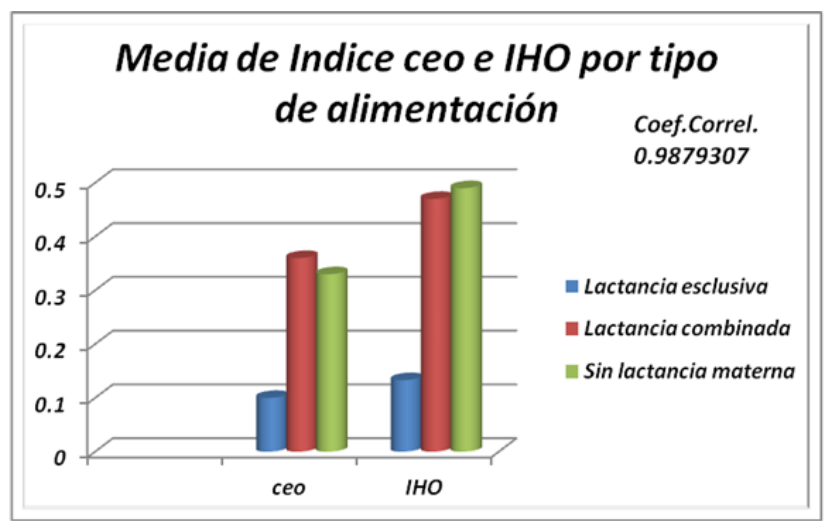

Figura 1. Distribución de Indice de caries e higiene oral por tipo de alimentación.

Figure 1. Distribution Index caries and oral hygiene by food type.

La distribución por estratos de los menores examinados, permite observar que la población alimentada al seno materno fue la menor de los tres grupos (Tabla 1).

Al comparar el índice de caries de acuerdo al tipo de alimentación implementada por la madre, se manifiesta que aquellos que recibieron lactancia materna exclusivamente por un periodo mayor de tiempo, presentaron un menor índice de caries (Figura 1).

Al observar el porcentaje de menores libres de caries, se puede constatar un mayor porcentaje en el grupo que recibio lactancia materna exclusiva (Figura 2).

El índice de higiene oral simplificado adaptado a la dentición primaria que se obtuvo en los tres grupos fue muy parecido $(0.3-0.6)$ con una media de 0.5 , ubicando a los niños examinados en el rango de buena higiene.

El CPO e IHOS de los padres de acuerdo al máximo grado académico alcanzado al momento de realizar el estudio ubican a los padres con un nivel de secundaria hasta universidad en un índice CPO moderado con una higiene regular y a los solo obtuvieron un nivel de estudios de primaria se ubicarón en un CPO Alto con una higiene oral mala (Figura 3).

Al comparar la relación entre el índice CPO de los padres con el índice ceo de los menores exa- 


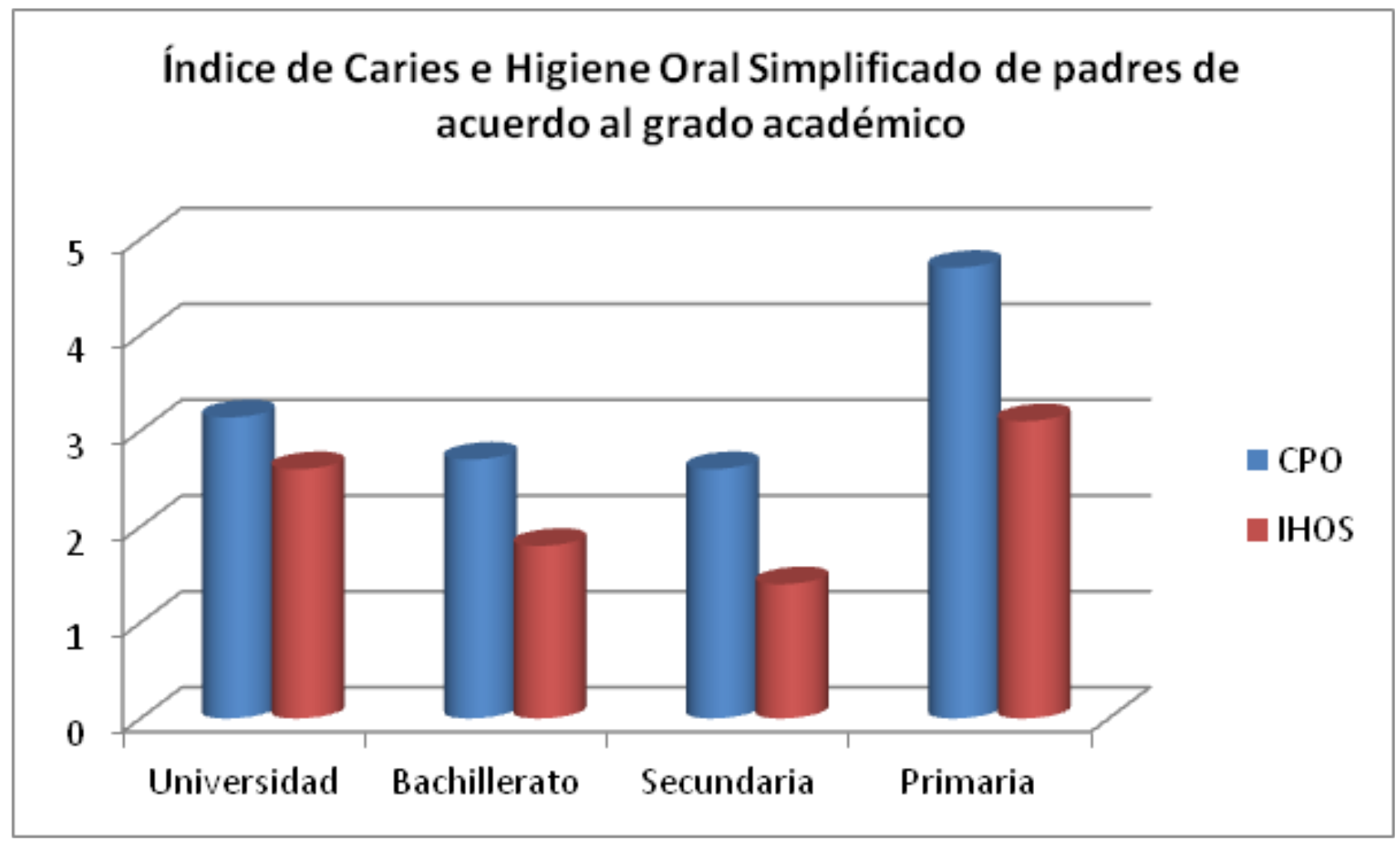

Figura 3. Descripción del Índice de Caries a Higiene Oral Simplificado de padres de niños examinados de acuerdo al máximo grado académico alcanzado a la fecha del estudio.

Figure 3. Description Caries Index Simplified Oral Hygiene parents of children examined according to the highest academic level reached at the time of the study.

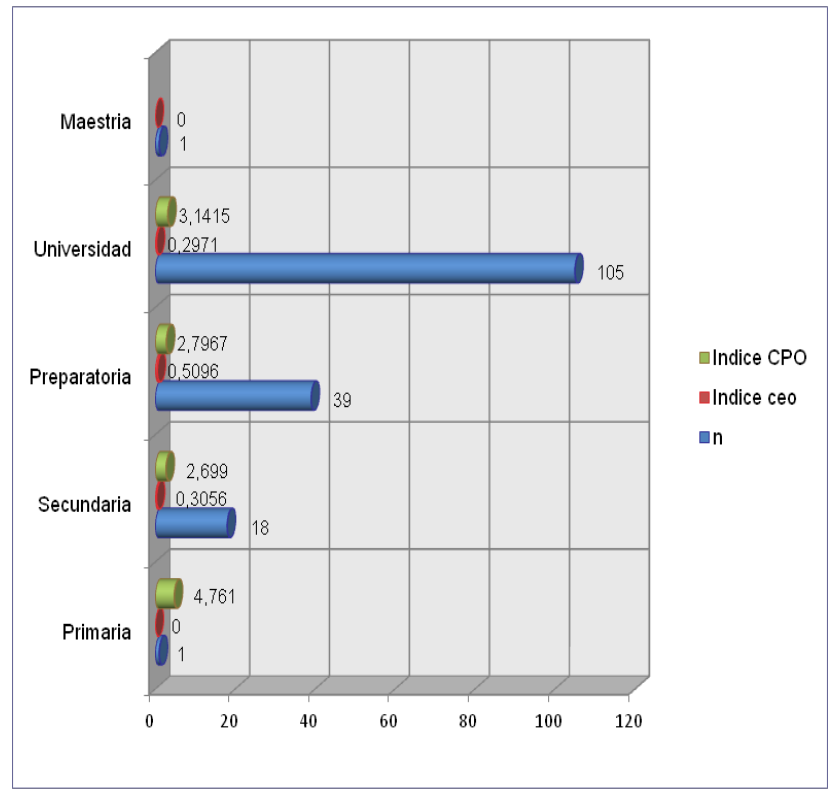

Figura 4. Escolaridad del padre, madre o tutor, su Índice CPO y el índice ceo de los menores examinados.

Figure 4. Schooling parent or guardian, the DMF index and dmf index of children examined. minados de acuerdo al grado de escolaridad de los padres, no se encontró asociación (Figura 4). La Tabla 2 muestra la media de las variables estudiadas y su relación con el índice de caries del menor, revelando la existencia de una relación entre ellas.

Con los datos ordenados al aplicar la prueba estadística de correlación de Pearson entre el patrón de alimentación y el índice ceo del menor, se obtuvo una correlación fuerte y perfecta entre el periodo de lactancia materna exclusivo en los 10 menores registrados e individuos libres de caries, encontrando una diferencia estadísticamente significativa (.000) (Tabla 3).

\section{Discusión}

De acuerdo a la Organización Mundial de la Salud, los valores del índice ceo muy bajo, van de 0 a 1.1, es claro que dentro de la escala de me- 


\begin{tabular}{cccc}
$\begin{array}{c}\text { Variables } \\
\text { de estudio }\end{array}$ & $\mathrm{N}$ & Media & $\begin{array}{c}\text { Desviación } \\
\text { típica }\end{array}$ \\
$\begin{array}{c}\text { Lactancia } \\
\text { materna } \\
\text { exclusiva }\left(^{*}\right)\end{array}$ & & $11\left(^{*}\right)$ & 7,453 \\
$\begin{array}{c}\text { Alimentación } \\
\text { complementaria( }\end{array}$ & 10 & $4.7\left(^{*}\right)$ & 1,059 \\
\hline $\begin{array}{c}\text { Incide ceo } \\
\text { Lactancia materna } \\
\text { combinada con } \\
\text { biberón }\left(^{*}\right)\end{array}$ & & 0,10 & 0,316 \\
\hline $\begin{array}{c}\text { Alimentación } \\
\text { complementaria }\left(^{*}\right)\end{array}$ & 121 & $5,1\left(^{*}\right)$ & 0,3684 \\
\hline Incide ceo & & 0,368 & 1,078 \\
\hline $\begin{array}{c}\text { Sin Lactancia } \\
\text { materna }\left(^{*}\right)\end{array}$ & & $0\left(^{*}\right)$ & $0\left(^{*}\right)$ \\
\hline $\begin{array}{c}\text { Alimentación } \\
\text { complementaria }\left(^{*}\right)\end{array}$ & 33 & $5,57\left(^{*}\right)$ & 3,544 \\
\hline $\begin{array}{c}\text { Incide ceo } \\
\text { Indice de Higiene } \\
\text { oral simplificado del } \\
\text { menor (IHOS) }\end{array}$ & 164 & 0,460 & 0,706 \\
\hline
\end{tabular}

\section{(*) Duración en meses}

Tabla 2. Medias de las variables del estudio en relación al indice ceo del menor.

Table 2. Means of the study variables in relation to the index dmf child.

dición del índice existe un límite superior y un límite inferior. Los resultados obtenidos en esta investigación indican que tanto en el GRUPO 2 (Lactancia materna y alimentación con biberón) como en el GRUPO 3 (Alimentados con biberón exclusivamente) evidencian una mayor presencia de lesiones cariosas en comparación al GRUPO 1 (Lactancia materna exclusiva), ubicando al GRUPO 1 más cercanamente al límite inferior el cual presenta un índice ceo de 0,10 sin embargo todos los grupos se encuentren dentro de la categoría de índice ceo muy bajo y no se encontró diferencia estadísticamente significativa entre los grupos de niños estudiados. Estos hallazgos coinciden parcialmente con lo publicado por AlDashti y col., (1995), por Gudiño y col., (2008) y Maldonado y col., (2010) que reportan una prevalencia de caries menor en niños con historia de

\begin{tabular}{|c|c|c|c|}
\hline & $\mathrm{N}$ & $\begin{array}{l}\text { Correlación } \\
\text { de Pearson }\end{array}$ & $\begin{array}{c}\text { Significancia } \\
\text { estadística }\end{array}$ \\
\hline \multirow[t]{2}{*}{$\begin{array}{l}\text { P. Lactancia } \\
\text { materna y el } \\
\text { índice ceo }\end{array}$} & & $0,896\left(^{*}\right)$ & 0,00 \\
\hline & 10 & & \\
\hline $\begin{array}{l}\text { Alimentación } \\
\text { complementaria } \\
\text { y el índice ceo }\end{array}$ & & 0,100 & 0,784 \\
\hline $\begin{array}{l}\text { P. Lactancia } \\
\text { materna } \\
\text { combinada } \\
\text { con biberón y } \\
\text { el índice ceo }\end{array}$ & & 0,71 & 0,437 \\
\hline
\end{tabular}

121

\begin{tabular}{|c|c|c|c|}
\hline $\begin{array}{l}\text { Alimentación } \\
\text { complementaria } \\
\text { y el índice ceo }\end{array}$ & & 0,139 & 0,130 \\
\hline $\begin{array}{l}\text { Alimentación } \\
\text { complementaria } \\
\text { y el índice ceo }\end{array}$ & 33 & 0,10 & 0,954 \\
\hline \multirow[t]{2}{*}{ Índice CPO } & 77 & & \\
\hline & & 0,10 & 0,933 \\
\hline Índice ceo & 164 & & \\
\hline
\end{tabular}

\section{(*) Correlación fuerte y perfecta.}

Tabla 3. Correlación de Pearson de las variables del estudio con el indice ceo.

Table 3. Pearson correlation of the study variables with the ceo index.

lactancia materna. Este dato puede ser explicado por los estudios de Erickson y col., (1999) quienes afirman que la leche materna no causa la descalcificación del esmalte. Es importante señalar que la leche materna contiene proteínas como la caseína (Reyolds 1987) que se une a la hidroxiapatita reduciendo su solubilidad y dificultando que los Streptococcus Mutans se adhieran a la superficie del diente. Además la leche materna, brinda una buena nutrición y contribuye a una menor ingesta de otros alimentos que pueden contener azúcares en su composición.

Al comparar los índices ceo de los menores examinados en el presente estudio (0.1 a 0.4$)$ con los índices ceo encontrados por la Secretaria de 
Salud en México (2.4 a 4.3) existe una diferencia muy marcada, es posible que ésta diferencia se deba a que los menores que participaron en éste estudio se encuentran en mejores condiciones por parte de sus cuidadores en las guarderías, este dato coincide con Pires dos Santos y col., (2002) y Alaluusua y col., (1994) ellos concluyen que la caries dental se encuentra relacionada con la presencia de placa dentobacteriana, en esta investigación, se detectó que los menores examinados independientemente de la práctica de alimentación, todos presentaron una buena higiene bucal, lo que contribuye a un menor número de lesiones cariosas. Debemos resaltar que las personas encargadas de los menores en las guarderías reciben pláticas sobre cuidados de la salud bucal y adiestramiento sobre técnicas de cepillado de acuerdo a la edad del menor, lo cual pudiera explicar los resultados y coincidir con lo reportado por los investigadores antes mencionados. En relación al desarrollo de la caries dental y factores secundarios asociados, se observó en la población examinada, que los hijos de padres que cursaron hasta la preparatoria, cuentan con la mayor cantidad de lesiones cariosas, en comparación con aquellos cuyos padres estudiaron hasta la universidad, éstos resultados coinciden con los estudios realizados por Rajab y col., (2002) y Mohebbi y col., (2006) ya que ellos señalan que la escolaridad más baja de los padres se relaciona con la mayor frecuencia de caries en los niños.

Uno de los hallazgos adicionales importantes de mencionar en esta investigación es que encontramos que el índice de caries de los menores examinados no muestra una correlación al índice de caries de los padres o tutores; éste hecho puede explicarse por la teoría de modificación del medio ambiente ya que los resultados de higiene demuestran muy pocos restos de alimentos adheridos a la superficie de los dientes de los menores examinados y este factor influye directamente en la cantidad de bacterias cariogénicas que pudieran colonizar dicha placa dental, por supuesto aquí también intervienen las personas de las guarderías encargadas de la higiene oral de los menores ya que reciben instrucción práctica de técnicas de cepillado de los dientes adecuadas a los menores, sin embargo, la caries dental es de naturaleza multifactorial en donde se encuentran relacionados otros factores como las características anatómicas de los dientes: la calidad de la estructura dental, cantidad o calidad de saliva y la exposición frecuente a carbohidratos fermentables debido al consumo de azucares, los cuales influyen en el desarrollo de caries dental y no solo el factor genético.

\section{Conclusiones}

De acuerdo al análisis de los resultados de la investigación podemos concluir que:

1. Los menores de 6 a 48 meses de edad que fueron alimentados con leche materna exclusivamente, se encontraron libres de caries.

2. La población estudiada en ésta investigación, se encuentra por debajo de los parámetros reportados por la secretaria de salud en México, ya que ellos reportaron índices ceo de 2,4 a 4,3 y en éste estudio se encontraron índices de 0,1 a 0,4 .

3. Solo el $6 \%$ de la población estudiada fue alimentado con leche materna de manera exclusiva, lo que ubica al resto, 94\%, con hábitos y costumbres alimenticios considerados de riesgo para caries dental, durante los primeros 6 meses de vida.

4. Existe una fuerte correlación entre el periodo prolongado de lactancia materna exclusivo y mantenerse libre de caries.

5. En cuanto al Índice de Higiene Oral Simplificado (IHOS) adaptado a dentición primaria, se identificó una buena higiene oral en la totalidad de la población examinada. 


\section{Referencias bibliográficas.}

1. Setiawati SF, Djoharanas H, Rina DR. Relationship between breastfeeding and early childhood caries (ECC) severity of children under three years old in DKI Jakarta. Makara, Kesehatan.2008; 12 (2): 87-92.

2. Guerrero CMP, Galeana RMG, Corona ZAA. Caries de la infancia temprana: medidas preventivas y rehabilitación. Rev Odont. Lat. 2011; 4(1):25-28.

3. Al-Dashti AA, Williams SA, Curzon ME. Breastfeeding, bottle feeding and dental caries in Kuwait, a country with lowfluoride levels in the water supply. 1995; (1): 42-7.

4. Alm A., Went LK, Koch G, Birkhed D, Nilsson M. Caries in adolescence - influence from early childhood. Community Dent Oral Epidemiol. 2012 40: 125-133.

5. Amarilla M, Quintero LG. Caries severa de aparición temprana: una nueva denominación para un antiguo problema. RAAO. 2006; 46 (2): 32- 5.

6. Favela OGF, Sanín RIP, Delgado MA. Caries de la infancia y sus factores de riesgo. AMOP. 2012; 24 (1): 11-15.

7. Gradella CMF, Bernabé E, Bönecker M, Oliveira LB. Caries prevalence and severity, and quality of life in Brazilian 2-to4year-old children. Community Dent Oral Epidemiol. 2011; 39: 498-504.

8. Gudiño FS. Caries de la temprana infancia: denominación, definición de caso y prevalencia en algunos países del mundo. UCR. 2006; 8: 39-45.

9. Maldonado RMA, Isassi HH, Padilla CJ. Diagnóstico temprano en problemas bucales. AMOP. 2008;20(2):24-27

10. Navarro AB, González A, Gil JF, Rioboo R. Caries del biberón. Rev Pediatr Aten Primaria. 1999;1 (4): 607-622.

11. Sánchez MJC, Sánchez Espejo F, Lomelí BG, Vera HH, Sanz BC, Mejía GA, Gaxiola C, Martínez RMA, Menchaca VO, Hernández MI, Mireles BA, Migoni IG, Escobar RME. 2009 Boletín Informativo SIVEPAB; 1: 1-8.

12. Sistema de Vigilancia Epidemiológica de Patologías Bucales SIVEPAB (2012). Serie en INTERNET 2013 [20 de Noviembre de 2013]; 1(1):1-73. Disponible en: www.epidemiologia.salud.gob.mx/SIVEPAB.

13. Dirección General de Información y Evaluación del Desempeño, Subsecretaria de Innovación y Calidad, Secretaria de Salud. Programa de Salud Bucal. México 2002; México DF: 124-5.

14. Gudiño FS. Lactancia materna, biberón, azúcares en solución y caries de la temprana infancia en el San José Urbano. Asociación Costarricense de congresos odontológicos. 2008

15. Arango MC, Baena GP. Caries de la infancia temprana y factores de riesgo. Rev.Estomat. $2004 ; 12$ (1): 59-65.

16. Kawashita Y, Kitamura M, Saito T. Early childhood caries. International Journal of Dentistry. 2011. Article ID 725320:1- 7.

17. Holm AK. Caries in the preschool child: International trends. J Dent. 1990; (18): 291-5.

18. Seow WK. Biological mechanisms of early childhood caries. Community Dent Oral Epidemiol.1998; 26(1): 8-27.

19. Alaluusua S, Malmivirta R. Early plaque accumulation--a sign for caries risk in

young children. 1994; Community Dent Oral Epidemiol.1994; (22): 273-6.

20. Maldonado RMA, Barrera GRA, Guzmán JRM, Hickman BM, Pantoja GV. Efecto de la dieta baja en carbohidratos sobre el pH salival en niños preescolares. AMOP. 2008;20(1):2-5.

21. UNICEF, OMS, UNESCO, UNFPA, PNUD, ONUSIDA, PMA y Banco Mundial. (2010) La nutrición y el crecimiento. Para la vida. 4ed. Nueva York, EU; 87.

22. Maldonado RMA, Torres BM, Issasi HH, Padilla CJ, Chávez PA, Reyes FR, Oliver PR. Lactancia materna: factor protector contra la caries dental. Rev Oral. 2010; 11 (33): 553-6.

23. Tsuguhiko Kato, Takashi Yorifuji, Michiyo Yamakawa, Sachiko Inoue, Keiko Saito, Hiroyuki Doi, Ichiro Kawachi.. Association of breast feeding with early childhood dental caries: Japanese population-based study. BMJ. 2015 (5):1-9. :e006982.

24. Davies GN. Early childhood caries- a synopsis. Community Dent Oral Epidemiol. 1998; (26): 106-16.

25. Duque ERJ, Pérez QJA, Hidalgo GFI. Caries dental y ecología bucal, aspectos importantes a considerar. Rev Cubana Estomatol. 2006; 43 (1): 1-11.

26. Dye, BA, Shenkin JD, Ogden LC, Marshall T, Levy SM, Kanellis MJ. The relationship between healtful eating practices and dental caries in children aged 2-5 years in the United States, 1988-1994. J Am Dent Assoc. 2004; (135): 55-66.

27. Erickson PR, Mazhari E. Investigation of the role of human breast milk in caries development. Pediatr Dent. 1999; $21: 86-90$.

28. Reynolds EC. The prevention of sub-surface demineralization of bovine enamel and change in plaque composition by casein in an intra-oral model. J Dent Res. 1987 Jun;66(6):1120-7.

29. Benjamin W. Chaffee and Ashley Cheng. Global Research Trends on Early-Life Feeding Practices and Early Childhood Caries: A Systematic Review. Journal of Oral Diseases Volume 2014: 1-7; Article ID 675658.

30. Birungi N, Fadnes LT, Okullo I, Kasangaki A, Nankabirwa V, Ndeezi G, et al. Effect of Breastfeeding Promotion on Early Childhood Caries and Breastfeeding Duration among 5 Year Old Children in Eastern Uganda: A Cluster Randomized Trial. PLoS ONE 2015 10(5): e0125352. doi:10.1371/journal.pone.0125352 
31. Hallet KB, Rourke KP. Early childhood caries and infant feeding practice. Community Dental Health. 2002 ; (19): $237-42$. 32. Harris R, Nicoll AD, Adair PM, Pine CM. Risk factors for dental caries in young children: a systematic review of the literature. Community Dent Health. 2004; 21:S71-85.

33. Matee M, Van't Hof M, Maselle S, Mikx F, Helderman WVP. Nursing caries, linear hypoplasia, and nursing and weaning habits in Tanzanian infants. Community Dent Oral Epidemiol. 1994; (22): 289-93.

34. Mohebbi SZ, Virtanen JI, Vahid-Golpayegani M, Vehkalahti MM. Early childhood caries and dental plaque among 1-3 year olds in Tehran, Iran. Journal Indian Soc Pedod Prev Dent. 2006;24(4):177-181.

35. Nunn ME, Braunstein NS, Krall KEA, Dietrich T, García RI, Henshaw MM. Health eating index is a predictor of early childhood caries. J Dent Res. 2009; 88(4): 361-6.

36. Pires dos Santos AP, Mendes SV. Caries prevalence and risk factors among children aged 0 to 36 months. Pesqui. Odontol. Bras. 2002;16 (3): 203-2008.

37. Rajab LD, Hamdan MAM. Early childhood caries and risk factors in Jordan. Community Dent Healt. 2002 ; (19): 224-9. 38. Rosemblatt A, Zarzar P. Prevalence of early childhood caries in 12 to 36 month-old children in Recife, Brazil. ASDC J Dent Child. 2002; (69): 319-24.

39. Tyagi R. The prevalence of nursing caries in Davangere preschool children and its relationship with feeding practices and socioeconomic status of the family. J Indian Soc Pedod Prevent Dent. 2008; (10): 153-7.

40. Vargas CM, Crall JJ, Schneider DA. Sociodemographic distribution of pediatric dental caries: NHANES III,1998-1994. JADA 1998; (129):1229-41.

41. Weerheijm KL, Uyttendaele-Speybrouck BFM, Euwe HC, Groen HJ. Prolonged demand breast- feeding and nursing caries. Caries Resp. 1998; (32): 46-50.

42. Yonezu T, Yotzuya K, Yakushiji M. Characteristics of breast-fed children with nursing caries. Bull Tokyo Dent Coll. 2006; 47 (4): 161-5.

Recibido: 06-01-2016

Aceptado: 03-10-2016

Correspondencia: Mario Alberto Maldonado Ramírez Boulevard Adolfo López Mateos \#100-C Altos Col. Unidad Nacional Cd. Madero, Tamaulipas. C.P. 89410. Tel. 833-2138247

E mail: odo.pediatrica.mario@gmail.com mmaldona@uat.edu.mx 